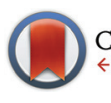

CrossMark

Cite this: Polym. Chem., 2017, 8, 641

\section{Janus nanoparticles inside polymeric materials: interfacial arrangement toward functional hybrid materials}

\author{
Qiuyan Yang and Katja Loos*
}

Received 13th October 2016, Accepted 16th November 2016 DOI: $10.1039 /$ c6py01795a www.rsc.org/polymers

\begin{abstract}
Control of the location and spatial organization of nanoparticles (NPs) inside polymers is essential to generate highly ordered NP-based functional devices including plasmonic waveguides, photonic crystals, optical lenses, memory storage devices, nanoelectronic circuits, photovoltaics, and batteries. Due to the unique combination of amphiphilicity and the particle character, Janus nanoparticles (JNPs) show high interfacial activity at fluid-fluid interfaces and in the bulk (for example, polymer blends and block copolymers (BCPS)). Interfacial incorporation of Janus NPs inside a polymeric matrix can endow polymeric materials with improved mechanical and additional properties from ordinary NPs. Here, different from other reports providing general overviews on the synthesis and applications of JNPs, this review specifically highlights recent advances and success in interfacial behavior of Janus NPs at polymer interfaces. We hope that these accomplishments will motivate additional efforts in large-scale synthesis and interfacial behavior studies of Janus NPs in polymer matrices allowing the design of functional hybrid nanostructures and devices with engineered, desired and tailored properties for real-life applications.
\end{abstract}

\section{Introduction}

Incorporating nanoparticles (NPs) into polymeric materials is not only a practical pathway to develop engineered plastics with increased mechanical, optical, electrical, magnetic, and other properties. This technique is also one of the most attractive ways to obtain well-defined structures at different length scales by controlling the spatial organization of NPs inside polymers. The term "polymer-nanoparticle nanocomposites" has already been applied since the early 20th century but just attracted broad interest until the $1990 \mathrm{~s} .{ }^{1,2}$ In the past 20 years, in order to be able to produce nanocomposites for practical use with engineered, desired, and tailored properties, extensive effort has been made to comprehensively understand the structure-property relationship in the polymer-NP mixture. ${ }^{3-13}$

One consensus is that control of the location and spatial organization of NPs inside polymers is essential for generating highly ordered NP-based functional devices including plasmonic waveguides, photonic crystals, optical lenses, memory storage devices, nanoelectronic circuits, photovoltaics, and batteries. ${ }^{2}$ Both experimental and theoretical results indicate that the dispersion and location of NPs inside polymers, block copolymers (BCPs) and polymer blends are governed by a deli-

Macromolecular Chemistry \& New Polymeric Materials, Zernike Institute for Advanced Materials, University of Groningen, Nijenborgh 4, 9747 AG Groningen, The Netherlands. E-mail: k.u.loos@rug.nl cate balance between enthalpic and entropic contributions, which rely on the properties of both polymer matrices (chemistry and rigidity) and particles (selectivity, size, and shape). Even though several general strategies based on the understanding of complex polymer-particle interaction have been proposed, precisely controlling the location of NPs in polymer matrices remains an obstacle in fabricating polymer-NP functional materials. $^{2}$

Janus NPs are defined as possessing different surface chemical/physical compositions on two sides of the NP and were initially named after the double-faced Roman god Janus (Fig. 1). Due to the unique combination of amphiphilicity and the particle character, Janus NPs are also reported to strongly adsorb and orient at the interface. Thus, their use shows

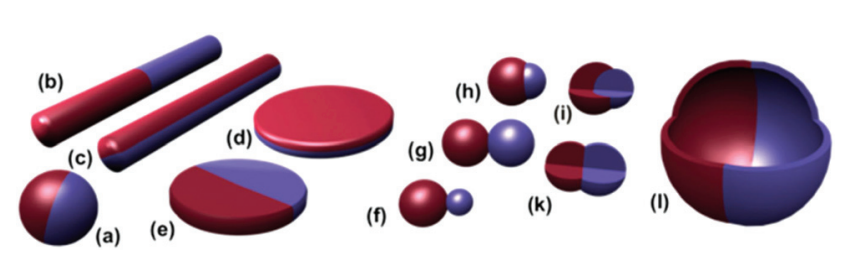

Fig. 1 Different types of Janus particles: spherical (a), two types of cylindrical (b, c), and disc-shaped (d, e) JPs. ( $f-k)$ Various kinds of dumbbell-shaped JPs with asymmetric or snowman character ( $f$ ), symmetric appearance ( $\mathrm{g}, \mathrm{k})$, attached nodes (h), and eccentric encapsulation (i). (l) Janus vesicles or capsules. Reprinted with permission from ref. 27. Copyright 2013 American Chemical Society. 
promise of achieving precise organization of multi-component NPs at the interface or surface of polymer matrices. These types of highly ordered hybrids have potential applications in flexible sensors, ${ }^{14-18}$ tunable plasmonic nanostructures for surface-enhanced Raman scattering, ${ }^{19-22}$ ultrafast switches and organic memory devices, ${ }^{23,24}$ and advanced photovoltaic devices. $^{25,26}$

This review will specifically focus on the theory and recent publications on the interfacial arrangement of NPs in polymeric materials. Our goal is to provide detailed information on how to obtain precise control of particle arrangement at the organic matrix interface in functional hybrid structures or devices.

In section 2, we summarize the synthesis and applications of Janus NPs in recent literature. Afterwards, the adsorption of Janus NPs at fluid-fluid interfaces is presented in order to obtain a better understanding of their interfacial behaviors as well, which is also helpful for fundamentally understanding how Janus NPs interact with polymeric interfaces, even though polymer interfaces cannot be simply understood as a fluidfluid interface and the flexibility and chemical properties of long-chain polymers should be considered as well.

In what follows, we present the latest studies on the interfacial location of Janus NPs inside polymer blends, in which Janus NPs show their superiority as compatibilizers for immiscible polymer blends.

In the last section, we describe the progress in the enthalpic and entropic effects for the interfacial location of NPs inside BCPs, especially Janus NPs. The interfacial behavior of various types of NPs based on their surface properties will be discussed in detail, including homogeneous NPs, ligand mixture coated NPs, random copolymer captured NPs and Janus NPs. The role of entropic effects (which rely on the Janus NP size, Janus NP shape, and chain properties of BCPs) in the orien- tation and off-center position at the interface will also be discussed, which is interesting for tailoring the band gaps of optical nano-composites.

\section{Synthesis of Janus nanoparticles}

Janus NPs are attractive materials for numerous applications $^{28-30}$ such as biological sensors, ${ }^{31,32}$ drug delivery, ${ }^{33-36}$ optical sensing devices, ${ }^{37-42}$ nano/ micromotors, ${ }^{43-46}$ two-phase stabilizers, ${ }^{47-49}$ and electronic displays. ${ }^{50-54}$ Due to the benefits of unique properties related to their asymmetric structure and/or functionalization, researchers from various fields have been attracted to investigating the preparation and properties of Janus NPs.

To date, a wide variety of techniques have been developed to produce NPs composed of both inorganic materials, such as ceramics, metals, oxides, salts, etc., and polymers. Considering that many reviews have already reported in detail on the fabrication of Janus NPs, ${ }^{27,55-61}$ a detailed comparison of these preparation methods would be out of the scope of this review.

Crucial issues in fabricating Janus materials, including high productivities and uniformities of asymmetric features, were mostly determined using the synthesis pathways. Synthesis approaches to zero-dimensional Janus materials with micro- or nano-structure can be generally categorized into the direct dual-supplied method and the indirect templateassisted method.

The direct dual-supplied method involves the formation of droplets consisting of two immiscible materials in a liquid or molten form. ${ }^{62}$ Biphasic particles with diameters of the order of tens of micrometers were then co-ejected via a spinning disk or a micro-fluidic system (Fig. 2a). ${ }^{63}$ The continuous ejection process in the direct dual-supplied method demonstrates

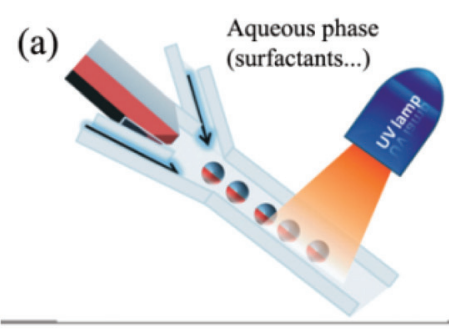

(b)

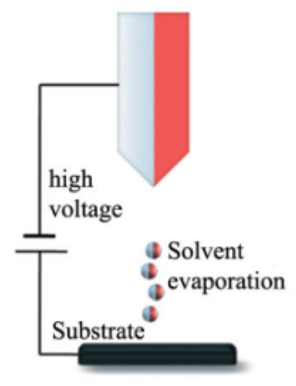

(c)

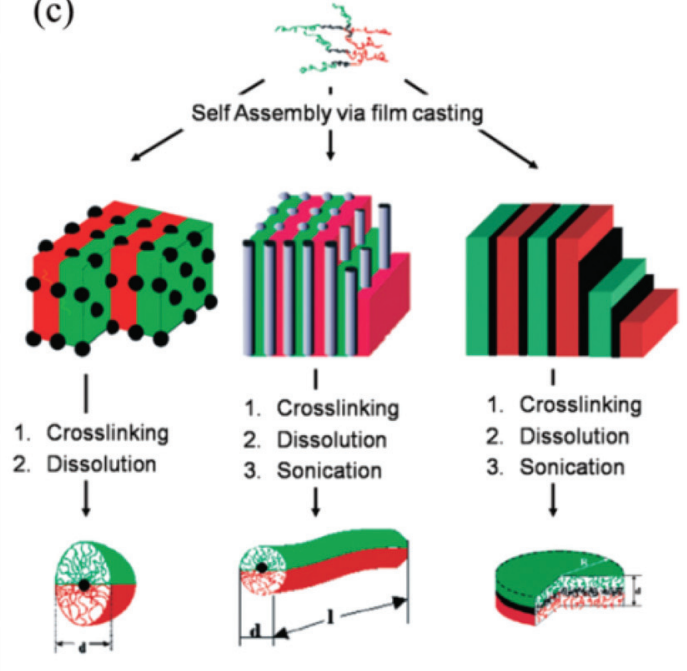

Fig. 2 Overview of direct preparation methods of Janus particles. (a) Micro-fluidic system; (b) electrospinning using a bi-phasic nozzle; (c) selfassembly of triblock terpolymers. Reprinted from ref. 60. Copyright the Royal Society of Chemistry 2008. 
the efficient production of Janus particles with moderate uniformities in terms of particle size and hemispheric features (Fig. 2b). ${ }^{64}$ Another direct preparation method of Janus particles is based on the self-assembly of block copolymers. Müller's group ${ }^{65-68}$ prepared cross-linked Janus polymer nanoparticles based on the self-organization of triblock terpolymers. This technique took advantage of the wide variety of complex morphologies (including micelles, ${ }^{66,69}$ cylinders, ${ }^{67,70,71}$ discs, ${ }^{68}$ etc.) with a high degree of spatial control that can be obtained spontaneously by the self-organization of terpolymers, depending on the chemical nature and molecular weights of the different blocks (Fig. 2c).

The indirect template-assisted method addresses the chemical or physical modifications of the hemispheric surfaces of existing mono-disperse particles. Particle embedding on substrate surfaces is required to conceal one hemispheric surface and to modify the other exposed hemisphere with chemical functionalities or geometric shapes. Particle adsorption and embedding are usually conducted on 2D flat surfaces (Fig. 3a). ${ }^{72-74}$ The major drawback of this strategy is that the amount of particles is extremely limited and does not allow their use in larger scale application studies. Nevertheless, due to its simplicity, this approach is still in use today.
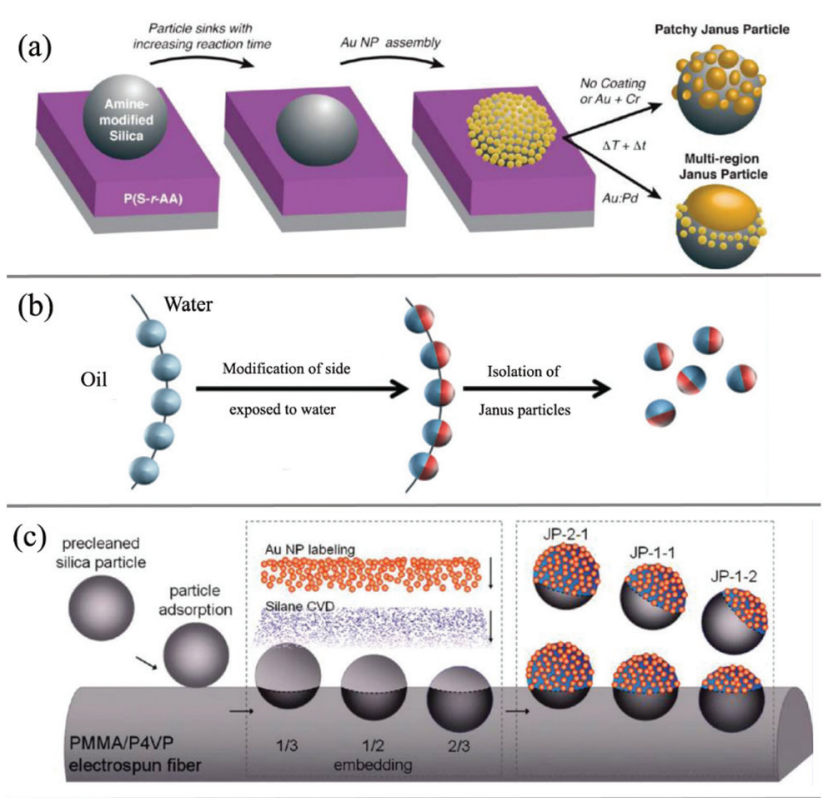

(d)



Fig. 3 Overview of indirect template-assisted methods of Janus particles. (a) 2D flat soft substrate. Reprinted with permission from ref. 72. Copyright 2010 American Chemical Society. (b) Liquid/liquid interface template. Reprinted with permission from ref. 60. Copyright the Royal Society of Chemistry 2008. (c) Fiber polymer substrate. Reprinted with permission from ref. 87. Copyright 2010 American Chemical Society. (d) Spherical polymer substrate. Reprinted with permission from ref. 89. Copyright the Royal Society of Chemistry 2013.
To overcome this limitation, spherical substrates offer an opportunity to increase the surface-to-volume ratio. For example, the effective fabrication of Janus silica particles has been demonstrated using suspended wax micro-particles as embedding vehicles by Granick and co-workers (Fig. 3b) ${ }^{75,76}$ The concept essentially transforms the two-dimensional technique into a solution phase and uses the high internal interface of an oil (wax)-water emulsion to achieve higher mass fractions of Janus particles. In a first step, they created a Pickering emulsion of wax and water using silica particles as stabilizers at high temperature. After cooling down of the emulsion and a purification step, the particles were immobilized at the solidified interface. The key step of this process is the immobilization of the particles at the interface and the suppressed rotational diffusion of the particles at the solidified interface. The Janus particles can then be obtained after functionalization of one side with aqueous phase chemistry and filtration at higher temperatures. This technique was subsequently used by other researchers to design various types of functional Janus NPs. ${ }^{77-82}$ For example, Okubo et al. have reported the formation of "mushroom-like" Janus particles from various polymer systems using this technique. ${ }^{83-86}$ Recently, Muller's group also synthesized hybrid silica Janus NPs with a poly(2-(dimethylamino)ethyl methacrylate) (PDMAEMA) hemicorona. Their Janus NPs show a reversible switching behavior upon $\mathrm{pH}$ and temperature changes and thus can self-assemble into linear strings at low $\mathrm{pH}$ and high concentration.

Another example of using polymer fiber as scaffolds for the immobilization of the particles to prepare Janus particles was presented by Kuo and coworkers (Fig. 3c). ${ }^{87,88}$ The authors reported the fabrication of binary and ternary Janus particles with even and uneven surface-functionalities by particle embedding and gas-phase silanization of the exposed surface.

In another approach (Fig. 3d), with the assistance of carbon dioxide $\left(\mathrm{CO}_{2}\right)$, polymer particles can be softened to allow a part of the rigid particle to be embedded inside their surface. With the embedded side being protected by a polymer substrate, further surface modification can be made on the other exposed side of rigid particles to form Janus-type NPs with subsequent removal of polymer templates by dissolving or thermal degradation. In this method, ${ }^{89,90}$ a spherical polymer template with a large surface-to-volume ratio allows largerscale production compared with 2D masking methods. Due to the benefits of their solid features after embedding, polymer substrates also demonstrate superior stability for particle spacing among templates compared to interface substrates suspended in liquid media. They can also simplify purification and separation with a liquid washing process, which is a great challenge for preparation methods based on immobilizing particles on a liquid/liquid or liquid/air interface. On the other hand, this method is quite suitable for preparing rigid inorganic or metal particles, but it may not be the best choice for other polymeric or "soft" Janus NPs, which can also be softened inside $\mathrm{CO}_{2}$. 
In brief, these abundant methods offer additional options to achieve Janus NPs. For example, Yabu et al. attempted different techniques to achieve organic-inorganic composite Janus NPs with various functional properties. ${ }^{91-95}$ But we should keep in mind that each of these approaches has its own merits and limitations, and people should choose the most suitable one to obtain the type of Janus NP for their own practical needs. ${ }^{27}$

\section{Interfacial properties of Janus nanoparticles at fluid-fluid interfaces}

The necessity to control and direct the self-assembly of NPs into defined superstructures arises from the need in materials and bio/life sciences to better exploit their often high intrinsic functionalities (e.g., electronic, mechanic, magnetic, conducting, and optic) for advanced materials. The ability to programme and reconfigure such structures so that they organize and restructure on demand is one of the ultimate goals. In this regard, external field-assisted alignment techniques may offer economical alternatives for fabricating highly ordered composite nanostructures. For example, various groups have already incorporated magnetic NPs or magnetic/metallic caps to investigate the self-assembly behavior of Janus NPs in magnetic ${ }^{96-103}$ or electric fields. ${ }^{104,105}$

A fluid-fluid interface is a natural platform for obtaining monolayer self-assembled structures of Janus NPs. Due to their high interfacial activity and amphiphilicity, Janus NPs can effectively lower the interfacial tension and thus strongly absorb and orient at the interface or surface, similarly to the mixed brush captured nanoparticles. ${ }^{106,107}$ One class of this interfacial location is the so-called Pickering effect of Janus NPs at the fluid-fluid or fluid-air interface, known as surfactant particles for emulsification. ${ }^{4-49}$ De Gennes, who called attention to Janus NPs in his Nobel Prize address, ${ }^{108}$ described the spontaneous monolayer arrangement from Janus NPs at, for instance, a water-air interface as a "breathable skin". He predicted the possibility of matter exchange between the two phases, which has recently been realized using Janus NPs as interfacial catalyst emulsifiers. ${ }^{109-112}$

The main reason why Janus NPs are able to stabilize emulsions and show their superior long-term stability more effectively than homogeneous particles can be explained by their strong adsorption energy to the fluid interface. Binks and Fletcher theoretically studied the energy to detach a single Janus NP from an oil-water interface, assuming a flat interface, as shown in Fig. $4 \mathrm{a} .{ }^{113}$ The total surface free energy $(E)$ for a Janus NP at the interface as a function of the angle $\beta$ in Fig. 4 is given by:

For $\beta \leq \alpha$

$$
\begin{aligned}
E(\beta)= & 2 \pi R^{2}[\gamma(\mathrm{AO})(1+\cos \alpha)+\gamma(\mathrm{PO})(\cos \beta-\cos \alpha) \\
& \left.+\gamma(\mathrm{PW})(1-\cos \beta)-\frac{1}{2} \gamma(\mathrm{OW})\left(\sin ^{2} \beta\right)\right]
\end{aligned}
$$
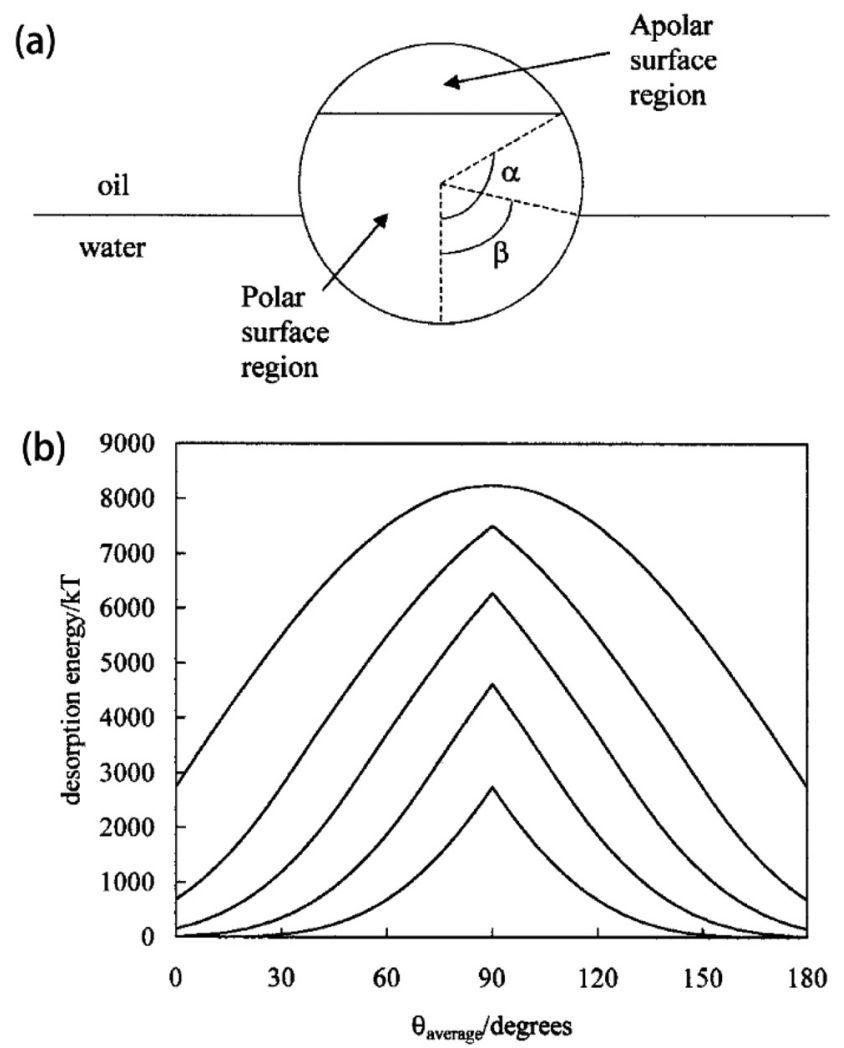

Fig. 4 (a) Geometry of a Janus particle at an oil-water interface with parameters $\alpha$ and $\beta$, which represent positions of the Janus boundary and the interface, respectively. (b) Variation of particle desorption energy with area-weighted average contact angle for particles of radius $10 \mathrm{~nm}$ and $\alpha=90^{\circ}$. Reprinted with permission from ref. 113. Copyright 2001 American Chemical Society.

For $\beta \geq \alpha$

$$
\begin{aligned}
E(\beta)= & 2 \pi R^{2}[\gamma(\mathrm{AO})(1+\cos \alpha)+\gamma(\mathrm{AW})(\cos \beta-\cos \alpha) \\
& \left.+\gamma(\mathrm{PW})(1-\cos \beta)-\frac{1}{2} \gamma(\mathrm{OW})\left(\sin ^{2} \beta\right)\right]
\end{aligned}
$$

where $R$ is the particle radius and $\gamma(\mathrm{AO}), \gamma(\mathrm{PO}), \gamma(\mathrm{AW}), \gamma(\mathrm{PW})$, and $\gamma(\mathrm{OW})$ are the interfacial energies of the apolar-oil, polaroil, apolar-water, polar-water, and oil-water interfaces, respectively. They weighted the average contact angle, plotted as the abscissa in Fig. 4a, by the relative areas of the polar and apolar particle surface regions according to:

$$
\theta_{\text {average }}=\frac{\theta_{\mathrm{A}}(1+\cos \alpha)+\theta_{\mathrm{P}}(1-\cos \alpha)}{2} .
$$

The amphiphilicity of Janus NPs can be "tuned" by the magnitude of the difference between the two contact angles $\theta_{\mathrm{A}}$ and $\theta_{\mathrm{P}}, \Delta \theta=\left(\theta_{\mathrm{P}}-\theta_{\mathrm{A}}\right) / 2$. Zero amphiphilicity (corresponding to homogeneous particles) corresponds to $\Delta \theta=0$. The strongest amphiphilicity is expected when $\Delta \theta=90^{\circ}$. According to their calculation of the desorption energy of a Janus NP for the fluid interface in Fig. 4b, it was shown that increasing the particle 
amphiphilicity through $\Delta \theta$ increases the strength of particle adsorption up to a maximum of three-fold for a $\theta_{\text {average }}$ of $90^{\circ}$. In addition, Janus NPs maintain their strong adsorption at average contact angles approaching 0 or $180^{\circ}$ where the surface activity of the non-amphiphilic (homogeneous with $\Delta \theta$ $=0$ ) particles is low. ${ }^{113}$ Further extensive theoretical simulations and experimental observations indicate that the interfacial activity of the Janus NPs can vary depending on several parameters, including the shape, size, morphology, and distribution of the spatial domains, and that the Janus NPs show an enhanced interfacial activity compared to the corresponding homogeneous particles, regardless of the synthesis and interfacial activity characterization methods. ${ }^{114-128}$

In analogy to the emulsification of fluid mixtures, Janus NPs are also expected to strongly attach to the interface inside polymer matrices, either in polymer blends or in block copolymers, which will be discussed in sections 3 and 4, respectively. A thorough study of the interfacial behavior of Janus NPs at the fluid-fluid interface is not only essential for further practical application of Janus NPs as solid stabilizers, but also helpful for fundamentally understanding how Janus NPs interact with polymeric interfaces, even though polymer interfaces cannot be simply understood as a fluid-fluid interface. The flexibility and chemical properties of long-chain polymers should be considered as well.

\section{Janus nanoparticles as compatibilizers for polymer blends}

Using molecular simulations, Estridge and Jayaraman ${ }^{129}$ compared the interfacial activity of different types of compatibilizers inside polymer blends, including diblock copolymer grafted nanoparticles (DBCGPs), BCPs, and Janus homopolymer grafted nanoparticles (JGPs). They showed that Janus NPs have the largest desorption energy due to their deeper penetration of the grafted beads into the A and $\mathrm{B}$ domain of the blend (Fig. 5a), and the lowest average interfacial tension of the compatibilized blend $(\gamma)$ normalized to the blend without compatibilizers $\left(\gamma_{0}\right)$ at all volume fractions of the compatibilizing agent $\phi$ considered (Fig. 5c). ${ }^{129}$ Unlike DBCGPs and BCPs, Janus NPs localized directly at the interface, allowing all the A (B) homopolymers grafted on one (other) hemisphere of the particle to interact with the A (B) domain of the blend (Fig. 5b) at all volume fractions of Janus NPs considered.

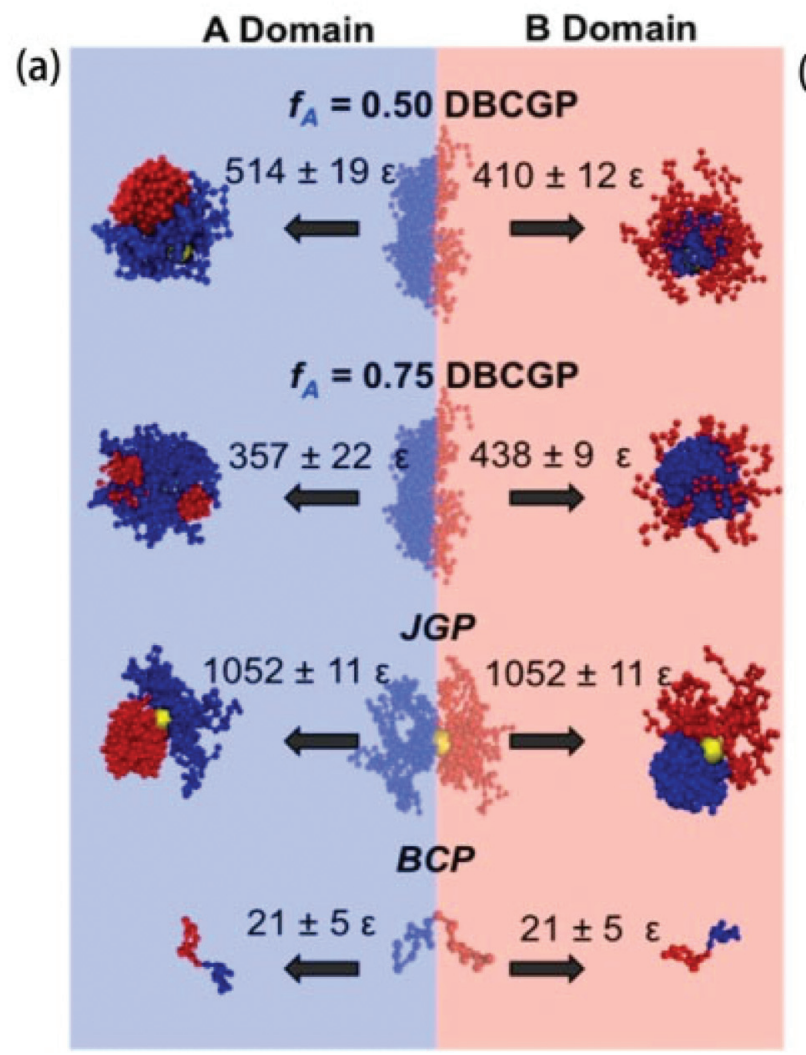

(b)

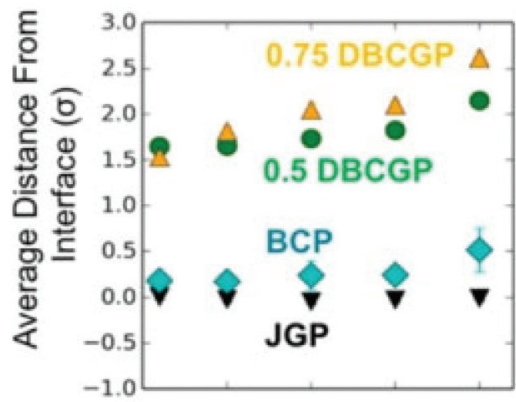

(c)

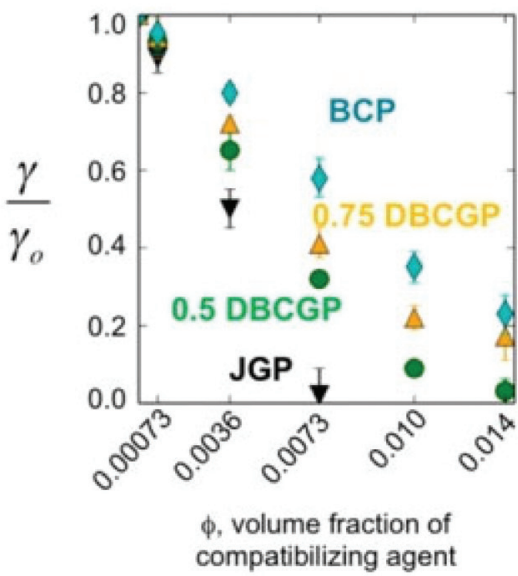

Fig. 5 (a) Energetic penalty for leaving the interface. These are representative snapshots of compatibilizer configurations at the interface and within each domain, and the desorption energies associated with each case. (b) Average distance of the compatibilizer from the interface versus volume fraction $\phi$ (the same $x$-axis as (c)). (c) Reduction in interfacial tension. Ratio of compatibilized blend interfacial tension ( $\gamma$ ) to compatibilizer-free blend interfacial tension $\left(\gamma_{0}\right)$ versus $\phi$. Error bars are standard error. Reprinted with permission from ref. 129. Copyright 2015 American Chemical Society. 
While a number of experimental and theoretical reports have demonstrated its advantages in improving the miscibility of immiscible binary polymers, homogeneous NPs still face complications in their use to stabilize polymer blends. Depending on variances in the surface chemistry, preferential wetting of homogeneous NPs inside one component can often occur. Interfacial adsorption can only be achieved if the difference between the interfacial tension values for the particles with each component is less than the interfacial tension of pure polymer blends themselves. ${ }^{130,131}$ Janus NPs, however,

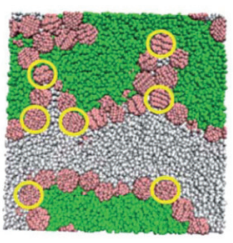

(a)

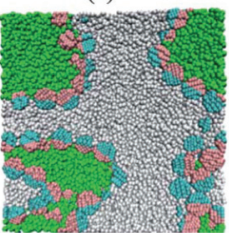

(b)

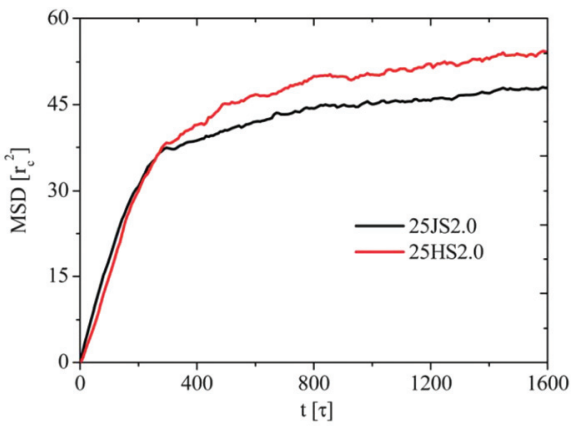

(c)
Fig. 6 Two regions selected from snapshots of $25 \mathrm{HS} 2.0$ (a) and $25 J$ S2.0 (b) systems at $t_{1 / 4}=1600 \mathrm{~s}$, which encompass a typical configuration that is seen throughout the samples. The yellow circle, which is drawn to guide the eye, outlines the nanoparticles that are not equatorially adsorbed at interfaces. The green, gray, pink, and cyan colors correspond to homopolymers A, homopolymers $\mathrm{B}$, and $\mathrm{p}$ and $\mathrm{q}$ hemispheres of Janus nanospheres, respectively. (c) The mean square displacement of nanospheres in the 25JS2.0 and 25HS2.0 systems. Reprinted with permission from ref. 132. Copyright the Royal Society of Chemistry 2012. are expected to exhibit a higher surface activity than homogeneous NPs and hence greatly reduce the interfacial tension to achieve interfacial location.

By taking the homogeneous NPs as a reference for comparison, numerical simulations by Guo and co-workers ${ }^{132,133}$ found that Janus nanospheres significantly hamper domain growth, and the average size of domains is smaller at later stages of the phase separation process (Fig. 6). Combining their other observations in ternary systems containing Janus NPs - including the slow domain growth at immediate and late times, crossover scaling domain growth behavior with the exponent close to 1 , and closer saturation of phase separation - they indicated that Janus nanospheres are equatorially adsorbed at interfaces and are not desorbed from the interface into the bulk. They concluded that Janus nanospheres can be used as a more effective emulsifying or stabilizing agent than homogeneous nanospheres for immiscible polymer blends.

More interestingly, by utilizing a Cahn-Hilliard model with Langevin equations, Krekhov et al. demonstrated that adding Janus particles above a certain concentration to a phase-separating binary mixture drives the system into regular structures with interfacially sequestered particles. Their simulation results propose a promising strategy to create periodic structures in binary mixtures by adding Janus particles. ${ }^{134}$

Experimentally, Virgilio and Favis investigated the effect of the affinity of Janus NPs and found that the Janus NPs consisting of polystyrene (PS) and poly(methyl methacrylate) (PMMA) spherical caps are interfacially active and locate at the highdensity polyethylene (HDPE) and polypropylene (PP) interface. ${ }^{135}$ Walther et al. ${ }^{136}$ also confirmed that organic Janus particles can be used to efficiently compatibilize polymer blends under high-shear conditions. Their results showed a constant decline of the domain size of the dispersed phase with the increased addition of Janus NPs, independent of the PS and PMMA blend composition. In the transmission electron microscopy (TEM) images (Fig. 7), Janus NPs were observed to

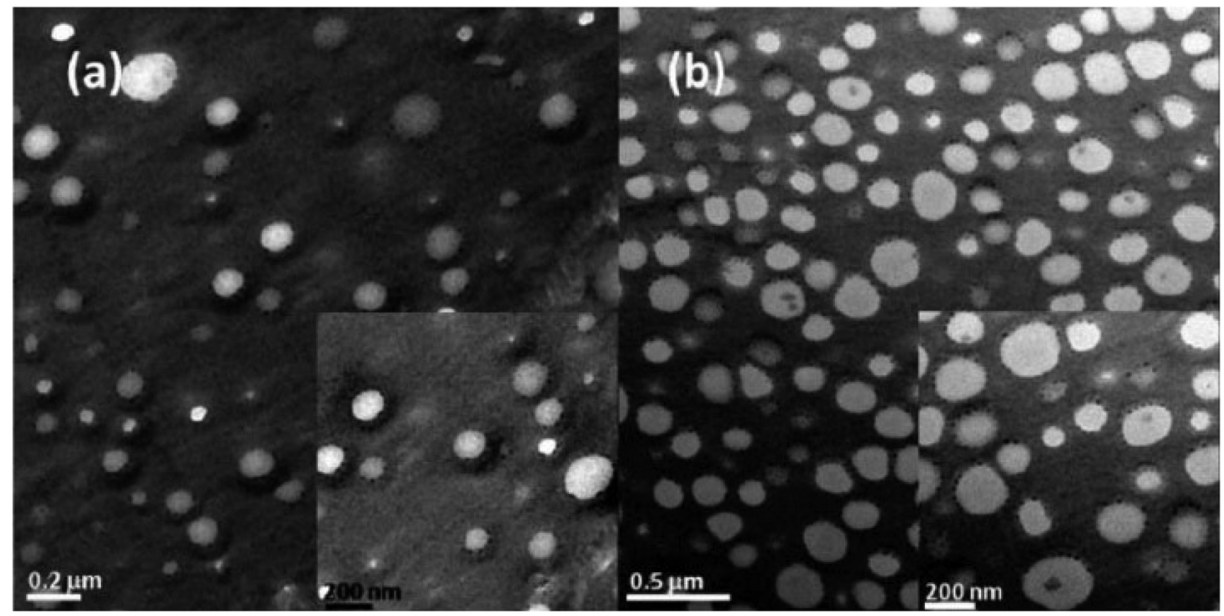

Fig. 7 TEM images of Janus particles and their adsorption at the blend interface of a PS/PMMA blend obtained for (a) 10 wt $\%$ JP in an $8 / 2$ PS/ PMMA blend and (b) $20 \mathrm{wt} \% \mathrm{JP}$ in a 6/4 PS/PMMA blend. Reproduced with permission from ref. 136. Copyright 2008 American Chemical Society. 
be almost exclusively located at the interface of the PS and PMMA domains, and only a negligibly small fraction was "lost" as unimers or micellar aggregates in one of the components. They considered this a significant improvement compared to BCP compatibilizers as a direct consequence of the high interfacial activity and increased adsorption energy at interfaces of Janus NPs. ${ }^{136}$ Janus-type kaolinite platelets obtained by Weiss et al. also showed an interfacial activity of Janus particles in PS/PMMA blends obtained by a solvent casting procedure. ${ }^{137}$

For industry-scale application, Bahrami et al. subsequently used $200 \mathrm{~g}$ of Janus NPs to compatibilize several kilograms of poly(2,6-dimethyl-1,4-phenylene ether) (PPE) and poly(styreneco-acrylonitrile) (SAN) (Fig. 8). ${ }^{138}$ The scaled-up experiments indicated that the fraction of Janus NPs in the range of 2-5 wt\% was the optimum amount necessary for sufficient droplet stabilization of PPE/SAN blends. The addition of only 0.5 and $1 \mathrm{wt} \%$ Janus NPs was not able to provide the necessary interface coverage and thus the blends were not mixed well. When a large amount of Janus NPs was added, excess content of Janus NPs inside the blends would create additional interfaces to form double emulsion morphologies. ${ }^{138}$ By using Janus NPs as the compatibilizer in the same polymer blend system, homogeneous polymeric foams with small cell sizes and relatively high densities are further produced with the plasticization of $\mathrm{CO}_{2}{ }^{139}$

Remarkably, due to the combination of strong interfacial affinity and the Pickering effect, Janus NPs were still quantitatively absorbed at the blend interface in these industry-scale blending experiments despite the harsh shear-processing conditions (Fig. 8). ${ }^{136,138}$ Soon afterwards, they also achieved a controlled blend morphology by de-mixing in solvent-cast films by adjusting the content of Janus NPs in blends and the composition ratio between PS and PMMA. ${ }^{140}$ Interfacially trapped structures in the final Janus NPs showed good resistance to coarsening during several days of annealing well above the glass transition temperature of the components. Similar phenomena from Janus NPs were also observed in other

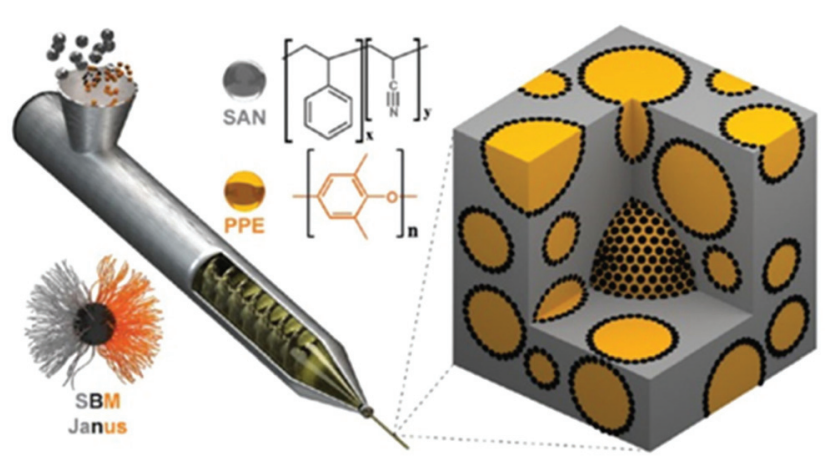

Fig. 8 Schematic representation of processing of polymer blends using JPs as a compatibilizer during extrusion of the polymer melt. JPs (black dots) compatibilize and stabilize PPE droplets (yellow) within the SAN matrix (gray). Reprinted with permission from ref. 138. Copyright 2014 American Chemical Society. different systems, such as immiscible poly(L-lactide) (PLLA)/ poly(vinylidene fluoride) (PVDF) blends, ${ }^{141,142}$ polystyrene/ polyamide- 6 blends, ${ }^{143}$ and polybutadiene/polyisoprene rubber blends. ${ }^{144}$

Janus NPs in the examples above show their superiority over other methods for compatibilizing immiscible polymer blends. They can mitigate the micellization problems of a diblock copolymer stabilizer and the difficulty of achieving interfacial adsorption encountered by homogeneous particle surfactants. As long as Janus inorganic particles can be prepared at the industrial scale, it would be quite promising to achieve hybrid materials with desired functional properties simply by mixing them with properly chosen polymer blends.

\section{Janus nanoparticles for ordered interfacial arrangement inside block copolymer scaffolds}

To generate highly ordered NP-based functional materials, control of the spatial organization of the NPs inside polymers is essential. The ability to control the length, spatial, and orientational organization of BCP morphologies (from spherical to cylindrical, bicontinuous, and lamellar structures) makes BCP materials particularly attractive as templates for manipulating the spatial location of inorganic NPs in various scales, from micro to nano. Several reviews have summarized the progress made in understanding and controlling NP distribution in BCP-ordered structures. ${ }^{1-13}$

Of particular interest is controlling the assembly of inorganic or metallic NPs, at the interface between different phase domains of the BCPs, to achieve a high degree of order and even responsive behaviors to certain external stimuli. ${ }^{145}$ However, interfacial location of NPs, and the overall morphology within BCPs, depends on a delicate balance between the enthalpy of NP insertion and the entropy related to particle conformation and translation, which are subjected to various interacting factors. These include the properties of BCPs (chemistry, conformation, and molecular weight) and of particles (selectivity, size, shape, and concentration).

\subsection{Enthalpic effects}

Enthalpic interactions rely on the surface chemistries of NPs and BCPs. With suitable grafting, polymeric ligands on NP surfaces can change the particle surface chemistry and effectively control NP-BCP enthalpic interactions and thus the location of NPs inside BCPs, either in one specific block domain or at the interface between blocks. For example, extensive studies were performed on the surface chemistry effect of NP grafting with polymeric ligands on their assemblies inside BCPs. It was shown that NPs, by modifying with A and B homopolymers, preferred to selectively localize within the $\mathrm{A}$ and $\mathrm{B}$ microdomains.

However, according to Kramer and his co-workers' observations, the surface coverage of polymer ligands on NPs can 
change this selectivity of NPs inside BCPs and, consequently, drive a shift in the spatial organization of NPs from inside the selective polymeric domain of the BCP matrix to the interfacial regions with the ligand areal density decrease. Polystyrenethiol (PS-SH) ligand-coated gold nanoparticles (Au NPs) have a critical areal chain density $\Sigma_{\mathrm{C}}$ below which NPs favorably adsorb to the interface. The $\Sigma_{\mathrm{C}}$ decreased from 3.1 to 0.9 chains per $\mathrm{nm}^{2}$ as the $M_{\mathrm{n}}$ of PS-SH chains increased from 1.5 to $13 \mathrm{~kg} \mathrm{~mol}{ }^{-1} \cdot{ }^{146-148}$

For the purpose of interfacial localization of NPs inside BCPs with better stability, the preferred choices are particles with amphiphilic surface properties, such as a mixture of ligands or random copolymer-anchored NPs and Janus-type NPs. Kim et al. reported that gold particles, which are coated with a mixture of low molecular weight PS and poly(2-vinyl pyridine) (P2VP) thiols, were observed to segregate at the interfaces between the PS-P2VP blocks over a broad range of PS fractions ( $\left.F_{\mathrm{PS}}\right)$ from 0.1 to 0.9 (Fig. 9). ${ }^{149,150}$

In stark contrast, Au NPs with surfaces covered by a random copolymer of styrene and 2-vinyl pyridine with $F_{\mathrm{PS}}=0.40$ remained in the P2VP domain and far away from the interface.
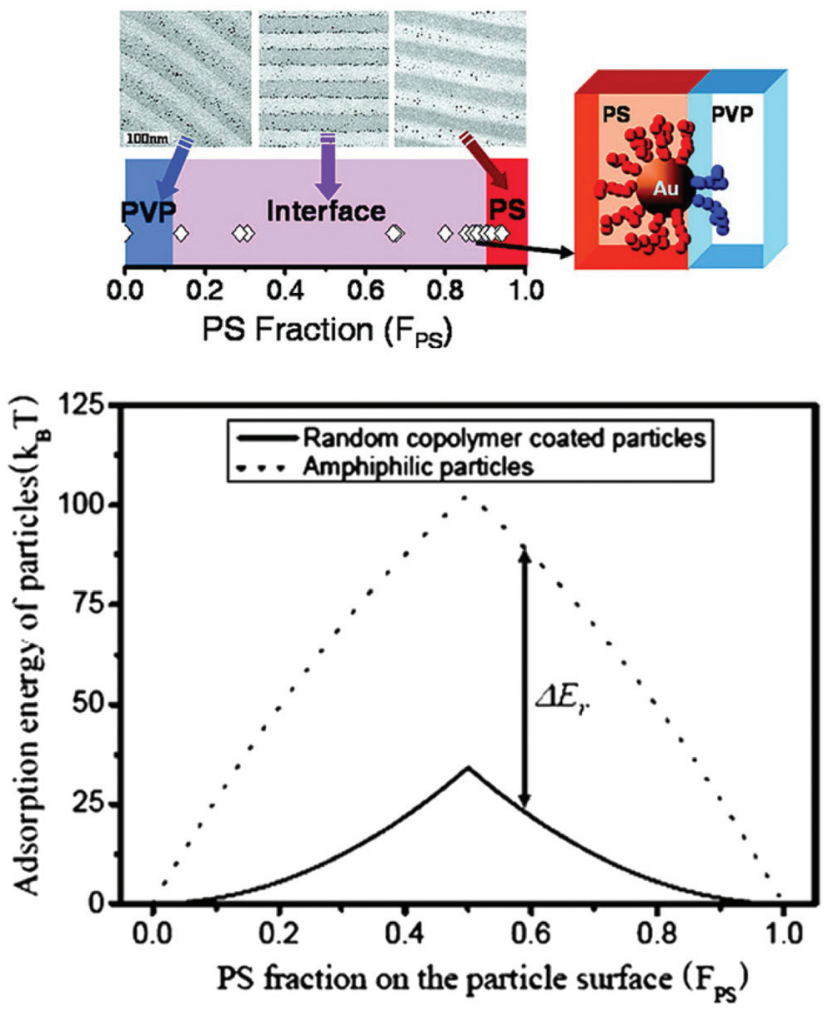

Fig. 9 (Upper) The spatial organization of Au NPs coated with a mixture of low molecular weight thiol end-functional PS/P2VP can be controlled in PS- $b$-P2VP. Varying the PS and P2VP surface compositions $\left(F_{\mathrm{PS}}\right)$ on the Au NPs allows placing the NPs into PS or P2VP microdomains or at the interface. (Lower) Adsorption energies for $4 \mathrm{~nm}$ radius random copolymer-coated NPs (solid line) and amphiphilic NPs (dotted line) are plotted as a function of $F_{\mathrm{PS}}$. Reprinted with permission from ref. 150. Copyright 2007 American Chemical Society.
However, random copolymer-coated Au NPs became clearly segregated along the PS/P2VP when the fraction increased to 0.52, which is consistent with the results in Fig. 9. ${ }^{150}$

Afterwards, Kim and Matsen developed a quantitative theoretical method to examine the effect of grafted brushes on the equilibrium distribution of spherical NPs inside the BCP lamellar phase by implementing self-consistent field theory and a new multi-coordinate-system scheme. ${ }^{151}$ Their simulation conclusions agreed with the experimental result that the mixed brushes are significantly more effective than the random-copolymer brushes at positioning NPs at the interface. However, the preference for the interface was much stronger than expected from simple surface-tension consideration, especially when mixture-grafted particles had a majority of PS chains on the surface $\left(F_{\mathrm{ps}}=0.9\right)$.

To explain the results, Kim et al. hypothesized that chains of the mixed brush segregate to opposite hemispheres, creating Janus NPs, as long as an appropriate time scale is involved. Once the particles adsorb at the interface, the ligands can rearrange on the AuNP surface, leading to strengthened adhesion and a pinning to the interface. They self-confirmed this possibility by their simple calculations on the adsorption energy of random copolymer-coated NPs to the interface (Fig. 9). ${ }^{150}$ Similar phenomena were also observed in polymer blends that interface by small-angle neutron scattering. It was revealed that two polymer ligands of high molecular weight showed phase separation to form a Janus-type shell on the NP surface, whereas those of low molecular weight formed either a mixed or partly de-mixed shell. ${ }^{152}$

The question arises, then, whether it is still necessary to use Janus NPs to capture interfaces, especially when mixed ligands or random copolymer-grafted NPs can do the same job and are even easier to prepare. The answer is affirmative for several reasons. First, as discussed in various examples above, ${ }^{129,149-151}$ Janus NPs theoretically show higher interfacial absorption energy than random copolymer-grafted NPs (or mixed polymer ligand-coated NPs without phase separation to form the Janus type) and thus are more preferentially located at the interface. Second, even though ligand exchanges on $\mathrm{Au}$ NPs are likely to form Janus-type particles at the polymer interface, it is not always true for other types of metallic or inorganic NPs, which endow hybrid materials with optical, magnetic, or electronic properties that Au NP-based hybrid materials cannot achieve. Actually, gold NPs with mixed ligands still were observed dispersing within one of the microdomains in PS- $b$-P2VP when the ratio of dodecanethiol to 1,1-mercapto-1-undecanol ligand was changed from 1:1 to $3: 1 .^{153}$ Also, coated with a ligand mixture with a molar ratio of approximately $5: 1$ of 1-dodecanethiol : PS-SH, Au NPs showed an affinity for PS-rich domains but did not localize at the interface in poly-(styrene- $b$-isoprene- $b$-styrene) triblock copolymer thin films. ${ }^{154}$ In these cases, the entropic effects surpassed enthalpic ones playing the main role in the location of NPs inside BCPs. However, limited by the rare experimental studies on the incorporation of Janus NPs into BCP scaffolds, it is difficult to draw a firm conclusion on which type of particle is 
better for interfacial location. To compare and thus reach a conclusion, more thorough studies on Janus NPs are needed.

\subsection{Entropic effects}

From the point of view of the enthalpy effect - that is, interaction between NPs and BCPs - Janus NPs with two chemically different grafting compartments tend to absorb at the interface inside BCPs. These benefit from their higher interfacial activity than homopolymer-coated NPs, and from their stronger amphiphilicity compared to homogeneous polymer-grafted NPs. However, their ordering and interface-centered position inside BCP composites is not simply determined by their surface chemistry, but usually governed by an intricate balance of enthalpic and entropic interactions. Therefore, entropic effects relying on the size, shape, and chain properties of BCPs should also be considered in designing new materials with regard to the orientation and off-center position at the interface.

5.2.1 Janus nanoparticle size. Wang et al. undertook an investigation on the effect of Janus NP size on NP distributions in BCP scaffolds, in the framework of self-consistent field theory/density functional theory (SCFT/DFT). They discovered that the Janus NPs' capability to stick to the interface is dependent on their sizes. Larger Janus NPs are found to be strongly attached to the interface with a higher value of the orientational order parameters. However, when the Janus NPs are smaller or one spherical cap becomes smaller than the other one, they are able to migrate from the interface to the domain of BCPs. ${ }^{155}$

It is remarkable to note that the conclusion on size-dependent interfacial location of Janus NPs in BCP scaffolds from Wang et al. ${ }^{155}$ seems opposite to that of homogeneous NPs inside BCPs addressed by both theory and modelling. ${ }^{148,156,157}$ For example, it was found that relatively smaller homogeneous particles (with the ratio of NP diameter, $d_{\mathrm{NP}}$, to the BCP domain size, $L$, being smaller than 0.2 ) were located in the interfacial regions of a polystyrene- $b$-poly(ethylene propylene) (PS- $b$-PEP) diblock copolymer, whereas relatively large NPs $\left(d_{\mathrm{NP}} / L>0.3\right)$ were localized in the interior of the PEP domains. ${ }^{2}$ There is, in fact, no conflict to observe completely different locations of larger Janus NPs and homogeneous NPs in the BCP matrix. Both systems indicate that favorable enthalpic interaction and minimal loss in the conformation entropy determine the particles' positions.

As an example, for larger particles, Janus types locate at the interface due to their amphipathicity and homogeneous ones are sequestered in the interior of one selective polymeric block. For smaller homogeneous particles, the stretching

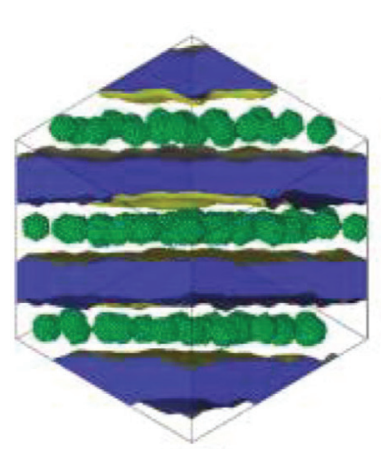

(a)

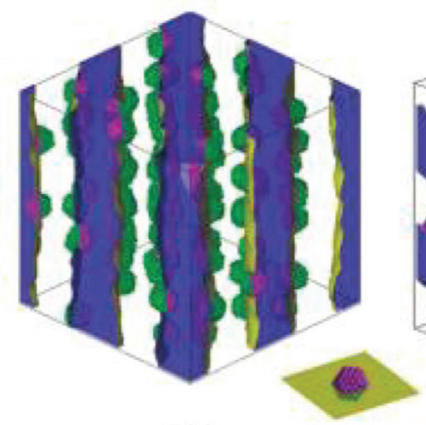

(b)

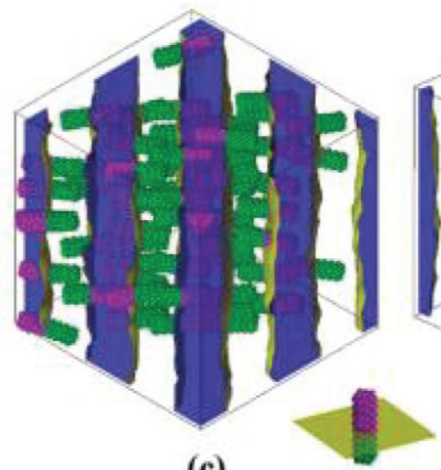

(c)

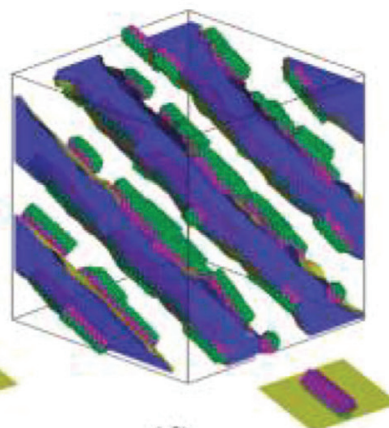

(d)

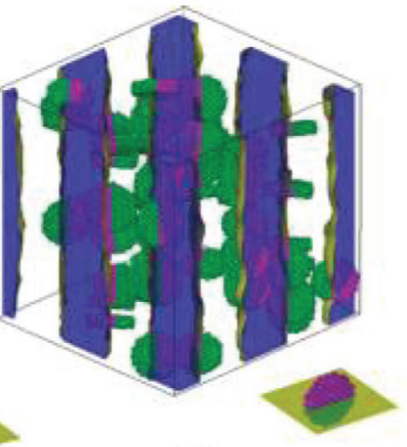

(e)

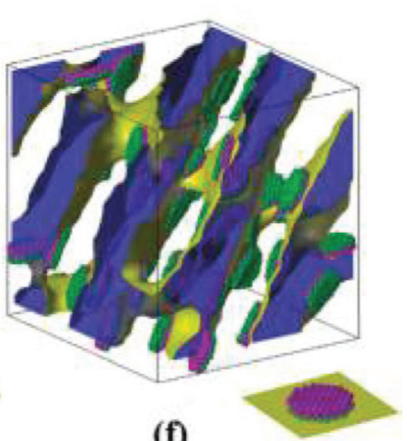

(f)



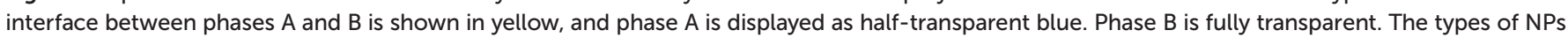

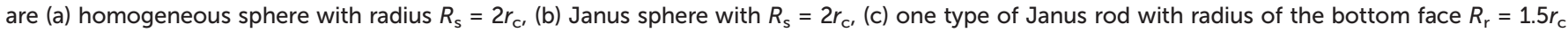

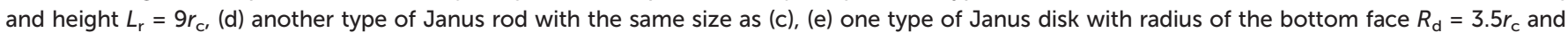

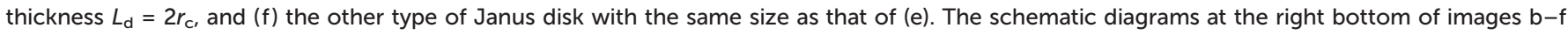

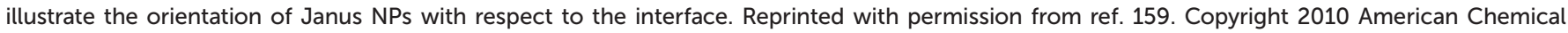
Society. 
required by the polymers to circumvent the spheres is less significant. Hence, it is the translational entropy of the particles that dominates the system's behavior. ${ }^{158}$ However, more reasonable theoretical explanations and experimental results are needed to explain the random distribution, but not interfacial arrangement, of smaller Janus NPs in BCP scaffolds.

5.2.2 Janus nanoparticle shape. Yan et al. conducted a simulation investigation on a mixture of a diblock copolymer and Janus NPs with different shapes. ${ }^{159}$ As shown in Fig. 10, Janus NPs, independent of their shapes, exclusively segregate to the interface between two lamellae at the equilibrium state. This reveals that the enthalpic effects from interactions between A and B blocks and the surface of Janus NPs overcome the entropic effects from the conformation transition of polymer chains under their simulation conditions. ${ }^{159}$ Benefiting from such strong enthalpic interactions between polymer blocks and the sphere surface, Janus spheres remain anchored in the interface during the whole dynamic assembling process of the nanocomposites and also during the dynamic shear process. ${ }^{159}$

It is interesting to note that the orientation of these anisotropic Janus NPs, with respect to the interface, can be controlled upon changing their surface and shape architectures, especially for Janus rods and disks. It was also suggested that Janus NP-containing diblock copolymers could allow better processing due to enhanced shear dynamics and feasible viscosity changes. ${ }^{159}$ Therefore, by using Janus-type NPs with surface and shape architectures, even more interfacial, stable, functional, and hybrid composites can be reached with oriented structures and responsive behaviors to certain external stimuli.

5.2.3 Block copolymer properties. In addition to the entropic effect caused by Janus NPs themselves, BCPs' properties can play an important role in the interfacial location of Janus NPs by influencing the polymer chain conformation entropy or particle translational entropy. To study the influence of BCP architecture on the precise position of Janus NPs with regard to the interface, systematic computer simulations and theoretical analyses were conducted by co-assembling symmetric Janus NPs into asymmetric diblock copolymer scaffolds of varying molecular architectures. This was done by simply increasing the length of the B block segment (while the length of the A block segment remained the same). ${ }^{160}$

For systems presenting a lamellar phase with flat-phase interfaces, Janus NPs tend to move to A domains for the longer

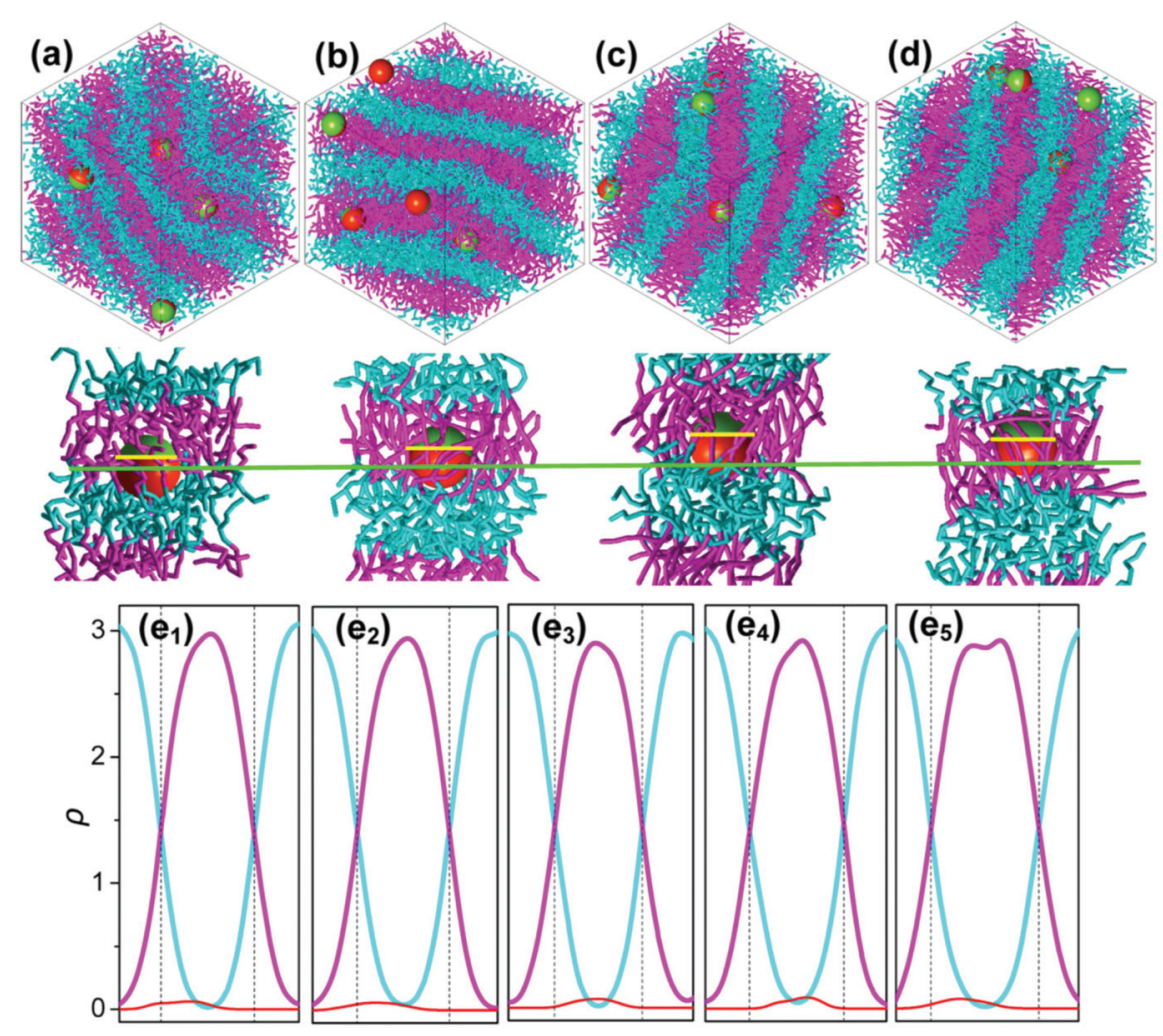

Fig. 11 (a-d) Representations of the self-assembly of Janus NPs in AB diblock copolymers with a flexible A block but with B block of various stiffnesses: (a) $K_{\mathrm{a}}=0 k_{\mathrm{B}} T$, (b) $K_{\mathrm{a}}=40 k_{\mathrm{B}} T$, (c) $K_{\mathrm{a}}=100 k_{\mathrm{B}} T$, and (d) $K_{\mathrm{a}}=300 k_{\mathrm{B}} T$. At each $K_{\mathrm{a}}$, the top snapshot shows the self-assembled morphologies where the cyan and pink chains denote blocks A and B, and sites P and Q of every Janus NP are shown in red and yellow, respectively. The bottom snapshot highlights the detailed position of a Janus NP at the interface and the conformation of the polymer chains around it. The green and yellow lines mark the phase interface and the equator of the NP $\left(e_{1}-e_{5}\right)$. The density profiles of blocks A (cyan) and B (pink) and the Janus NP (red) are shown for various stiffnesses of block B: $\left(\mathrm{e}_{1}\right) K_{\mathrm{a}}=5 k_{\mathrm{B}} T,\left(\mathrm{e}_{2}\right) K_{\mathrm{a}}=40 k_{\mathrm{B}} T,\left(\mathrm{e}_{3}\right) K_{\mathrm{a}}=60 k_{\mathrm{B}} T,\left(\mathrm{e}_{4}\right) K_{\mathrm{a}}=100 k_{\mathrm{B}} T$, and $\left(\mathrm{e}_{5}\right) K_{\mathrm{a}}=400 k_{\mathrm{B}} T$. Reprinted with permission from ref. 161. Copyright 2015 American Chemical Society. 
B segments, of which the stretched conformation makes it difficult for the Janus NPs to be positioned in the B segment. ${ }^{160}$ However, for even larger B segment systems, where the phase interface curves (BCP morphologies cylinder), the increase of B segments also causes Janus NPs to shift offcenter from the interface but turn to the B domains. ${ }^{160}$ In this case, the topology mismatching between Janus NPs and curved polymer interfaces at the meso-scale accounts for the Janus NPs' position transition. ${ }^{160}$ Similarly, the semiflexible block's stiffness can also regulate the off-center distribution of symmetrical Janus NPs with respect to the phase interface, featured by a roughly $35 \%$ deviation from the interface to the utmost extent (Fig. 11). ${ }^{161}$

\section{Summary and outlooks}

Studies on the interfacial behavior of Janus NPs at the fluidfluid interface are fundamental for understanding how Janus NPs interact with polymeric interfaces and thus designing hybrid materials. Based on the theoretical simulations and experimental studies, Janus NPs show their superiority over other methods for compatibilizing, regardless of the harsh processing conditions.

For the purpose of interfacial localization of NPs inside BCPs with better stability, particles with amphiphilic surface properties are the preferred choices, including mixtures of ligands or random copolymer-anchored NPs and Janus-type NPs. Compared with random copolymer-grafted NPs and mixed polymer ligand-anchored NPs without phase separation, Janus NPs show higher interface activity and amphipathicity. They were observed still remaining in the block interface region even during shearing, independent of their shape, and therefore hybrid material with higher performance properties could be expected. Entropic effects rely on the size, shape, and chain properties of BCPs, but also play an important role in the orientation and off-center position at the interface, which is interesting for tailoring the band gaps of optical nanocomposites.

This type of hybrid materials based on the precise location of Janus NPs at the interface in polymeric materials can combine the functional properties of NPs with the mechanical properties of polymer matrices. With properly choosing the type of Janus NP and polymer, one therefore can flexibly design functional hybrid nanostructures and devices with engineered, desired, and tailored properties toward real-life applications.

Despite these theoretical studies, establishing more detailed knowledge regarding the effect of Janus NPs on their positions in BCP scaffolds is still needed to create nanocomposites with desired structures: especially an experimental study, which is still rare, perhaps due to the more complicated synthesis required. Motivating additional efforts in large-scale synthesis and interfacial behavior studies of Janus NPs for the design of these smart materials is actually one of the main purposes of this review.

\section{Abbreviations}

$\begin{array}{ll}\mathrm{NP} & \text { Nanoparticle } \\ \mathrm{BCP} & \text { Block copolymer } \\ \mathrm{CO}_{2} & \text { Carbon dioxide } \\ \mathrm{AO} & \text { Apolar-oil } \\ \mathrm{PO} & \text { Polar-oil } \\ \mathrm{AW} & \text { Apolar-water } \\ \mathrm{PW} & \text { Polar-water } \\ \mathrm{OW} & \text { Oil-water } \\ \text { DBCGPS } & \text { Diblock copolymer grafted nanoparticles } \\ \text { PS } & \text { Polystyrene } \\ \text { PMMA } & \text { Poly(methyl methacrylate) } \\ \mathrm{HDPE} & \text { High-density polyethylene } \\ \text { PP } & \text { Polypropylene } \\ \text { TEM } & \text { Transmission electron microscopy } \\ \text { PPE } & \text { Poly(2,6-dimethyl-1,4-phenylene ether) } \\ \text { SAN } & \text { Poly(styrene-co-acrylonitrile) } \\ \text { PS-SH } & \text { Polystyrene-thiol } \\ \text { Au NP } & \text { Gold nanoparticle } \\ \text { P2VP } & \text { Poly(2-vinyl pyridine) } \\ \text { SCFT/ } & \text { Self-consistent field theory/density functional } \\ \text { DFT } & \text { theory } \\ \text { PS- } b \text {-PEP } & \text { Polystyrene- } b \text {-poly(ethylene propylene) } \\ \text { PEP } & \text { Poly(ethylene propylene) } \\ & \end{array}$

\section{Acknowledgements}

The authors acknowledge funding support from the China Scholarship Council and the Netherlands Organisation for Scientific Research (NWO).

\section{References}

1 A. C. Balazs, T. Emrick and T. P. Russell, Science, 2006, 314, 1107-1110.

2 B. Sarkar and P. Alexandridis, Prog. Polym. Sci., 2015, 40, 33-62.

3 J. Huh, V. V. Ginzburg and A. C. Balazs, Macromolecules, 2000, 33, 8085-8096.

4 R. A. Vaia and J. F. Maguire, Chem. Mater., 2007, 19, 27362751.

5 M. R. Bockstaller, R. A. Mickiewicz and E. L. Thomas, $A d v$. Mater., 2005, 17, 1331-1349.

6 R. Shenhar, T. B. Norsten and V. M. Rotello, Adv. Mater., 2005, 17, 657-669.

7 A. Haryono and W. H. Binder, Small, 2006, 2, 600-611.

8 A. Fahmi, T. Pietsch, C. Mendoza and N. Cheval, Mater. Today, 2009, 12, 44-50.

9 M. Grzelczak, J. Vermant, E. M. Furst and L. M. LizMarzán, ACS Nano, 2010, 4, 3591-3605.

10 K. Galatsis, K. L. Wang, M. Ozkan, C. S. Ozkan, Y. Huang, J. P. Chang, H. G. Monbouquette, Y. Chen, P. Nealey and Y. Botros, Adv. Mater., 2010, 22, 769-778. 
11 H.-C. Kim, S.-M. Park and W. D. Hinsberg, Chem. Rev., 2010, 110, 146-177.

12 H. Zhang, Y. Liu, D. Yao and B. Yang, Chem. Soc. Rev., 2012, 41, 6066-6088.

13 J. Kao, K. Thorkelsson, P. Bai, B. J. Rancatore and T. Xu, Chem. Soc. Rev., 2013, 42, 2654-2678.

14 M. Segev-Bar and H. Haick, ACS Nano, 2013, 7, 8366-8378.

15 B. Yoon, W. D. Luedtke, R. N. Barnett, J. P. Gao, A. Desireddy, B. E. Conn, T. Bigioni and U. Landman, Nat. Mater., 2014, 13, 807-811.

16 L. Z. Yi, W. H. Jiao, K. Wu, L. H. Qian, X. X. Yu, Q. Xia, K. M. Mao, S. L. Yuan, S. Wang and Y. T. Jiang, Nano Res., 2015, 8, 2978-2987.

17 N. Kahn, O. Lavie, M. Paz, Y. Segev and H. Haick, Nano Lett., 2015, 15, 7023-7028.

18 W. Zhao, J. Luo, S. Y. Shan, J. P. Lombardi, Y. Xu, K. Cartwright, S. Lu, M. Poliks and C. J. Zhong, Small, 2015, 11, 4509-4516.

19 I. Tokarev and S. Minko, Soft Matter, 2012, 8, 5980-5987.

20 Y.-G. He, S.-Y. Shi, N. Liu, Y.-Y. Zhu, Y.-S. Ding, J. Yin and Z.-Q. Wu, RSC Adv., 2015, 5, 39697-39704.

21 S. Fateixa, H. I. S. Nogueira and T. Trindade, Phys. Chem. Chem. Phys., 2015, 17, 21046-21071.

22 A. X. Wang and X. M. Kong, Materials, 2015, 8, 3024-3052.

23 W.-P. Lin, S.-J. Liu, T. Gong, Q. Zhao and W. Huang, Adv. Mater., 2014, 26, 570-606.

24 J. Y. Ouyang, J. Mater. Chem. C, 2015, 3, 7243-7261.

25 G. M. Kang, J. Yoo, J. Ahn and K. Kim, Nano Today, 2015, 10, 22-47.

26 G. Li, R. Zhu and Y. Yang, Nat. Photonics, 2012, 6, 153-161.

27 A. Walther and A. H. E. Müller, Chem. Rev., 2013, 113, 5194-5261.

28 A. Synytska, R. Khanum, L. Ionov, C. Cherif and C. Bellmann, ACS Appl. Mater. Interfaces, 2011, 3, 12161220.

29 H. Xing, Z. D. Wang, Z. D. Xu, N. Y. Wong, Y. Xiang, G. L. Liu and Y. Lu, ACS Nano, 2012, 6, 802-809.

30 S.-H. Kim, S. Y. Lee and S.-M. Yang, Angew. Chem., Int. Ed., 2010, 49, 2535-2538.

31 J. L. Tang, K. Schoenwald, D. Potter, D. White and T. Sulchek, Langmuir, 2012, 28, 10033-10039.

32 M. Yoshida, K. H. Roh, S. Mandal, S. Bhaskar, D. W. Lim, H. Nandivada, X. P. Deng and J. Lahann, Adv. Mater., 2009, 21, 4920-4925.

33 C. Kaewsaneha, P. Tangboriboonrat, D. Polpanich, M. Eissa and A. Elaissari, ACS Appl. Mater. Interfaces, 2013, 5, 1857-1869.

34 L.-T.-C. Tran, S. Lesieur and V. Faivre, Expert Opin. Drug Delivery, 2014, 11, 1064-1074.

35 I. Schick, S. Lorenz, D. Gehrig, S. Tenzer, W. Storc, K. Fischer, D. Strand, F. Laquai and W. Tremel, Beilstein J. Nanotechnol., 2014, 5, 2346-2362.

36 Y. J. Wu, X. K. Lin, Z. G. Wu, H. Möhwald and Q. He, ACS Appl. Mater. Interfaces, 2014, 6, 10476-10481.

37 H. H. Wang, S. Y. Yang, S.-N. Yin, L. Chen and S. Chen, ACS Appl. Mater. Interfaces, 2015, 7, 8827-8833.
38 S. Nedev, S. Carretero-Palacios, P. Kühler, T. Lohmüller, A. S. Urban, L. J. E. Anderson and J. Feldmann, ACS Photonics, 2015, 2, 491-496.

39 K. Miki, H. Hashimoto, T. Inoue, H. Matsuoka, H. Harada, M. Hiraoka and K. Ohe, Small, 2014, 10, 3119-3130.

40 J. H. Yoon, J. H. Lim and S. Yoon, ACS Nano, 2012, 6, 7199-7208.

41 Y. Duk Han, H.-S. Kim, Y. M. Park, H. J. Chun, J.-H. Kim and H. C. Yoon, ACS Appl. Mater. Interfaces, 2016, 8, 10767-10774.

42 A. J. Logsdail and R. L. Johnston, J. Phys. Chem. C, 2012, 116, 23616-23628.

43 M. Guix, C. C. Mayorga-Martinez and I. A. Merkoç, Chem. Rev., 2014, 114, 6285-6322.

44 X. Ma, A. Jannasch, U.-R. Albrecht, K. Hahn, A. MiguelLópez, E. Schäffer and S. Sánchez, Nano Lett., 2015, 15, 7043-7050.

45 W. Gao, A. Pei, R. F. Dong and J. Wang, J. Am. Chem. Soc., 2014, 136, 2276-2279.

46 W. Gao and J. Wang, ACS Nano, 2014, 8, 3170-3180.

47 A. Kumar, B. J. Park, F. Tu and D. Lee, Soft Matter, 2013, 9, 6604-6617.

48 L. Botto, E. P. Lewandowski, J. M. Cavallaro and K. J. Stebe, Soft Matter, 2012, 8, 9957-9971.

49 A. Böker, J. He, T. Emrick and T. P. Russell, Soft Matter, 2007, 3, 1231-1248.

50 Y. Komazaki, H. Hirama and T. Torii, J. Appl. Phys., 2015, 117, 154506.

51 A. M. Boymelgreen and T. A. Miloh, Phys. Fluids, 2011, 23, 072007.

52 Y. Daghighi and D. Q. Li, Lab Chip, 2011, 11, 2929-2940.

53 A. M. Boymelgreen and T. Miloh, Phys. Fluids, 2012, 24, 082003.

54 L. Zhang and Y. X. Zhu, Appl. Phys. Lett., 2010, 96, 141902.

55 X. Pang, C. Wan, M. Wang and Z. Lin, Angew. Chem., Int. Ed., 2014, 53, 5524-5538.

56 J. Reguera, H. Kim and F. Stellacci, Chimia, 2013, 67, 811818.

57 J. Hu, S. Zhou, Y. Sun, X. Fang and L. Wu, Chem. Soc. Rev., 2012, 41, 4356-4378.

58 B. T. T. Pham, C. H. Such and B. S. Hawkett, Polym. Chem., 2015, 6, 426-435.

59 M. Lattuadaa and T. A. Hattonb, Nano Today, 2011, 6, 286-308.

60 A. Walther and A. H. E. Müller, Soft Matter, 2008, 4, 663-668.

61 A. Perro, S. Reculusa, S. Ravaine, E. Bourgeat-Lami and E. Duguet, J. Mater. Chem., 2005, 15, 3745-3760.

62 G. Loget and A. Kuhn, J. Mater. Chem., 2012, 22, 1545715474.

63 K.-H. Roh, D. C. Martin and J. Lahann, Nat. Mater., 2005, 4, 759-763.

64 Z. Liu, D. D. Sun, P. Guo and J. O. Leckie, Nano Lett., 2007, 7, 1081-1085.

65 R. Erhardt, M. Zhang, A. Böker, H. Zettl, C. Abetz, P. Frederik, G. Krausch, V. Abetz and A. H. E. Müller, J. Am. Chem. Soc., 2003, 125, 3260-3267. 
66 R. Erhardt, A. Böker, H. Zettl, H. Kaya, W. PyckhoutHintzen, G. Krausch, V. Abetz and A. H. E. Müller, Macromolecules, 2001, 34, 1069-1075.

67 Y. Liu, V. Abetz and A. H. E. Müller, Macromolecules, 2003, 36, 7894-7898.

68 A. Walther, X. André, M. Drechsler, V. Abetz and A. H. E. Müller, J. Am. Chem. Soc., 2007, 129, 61876198.

69 A. H. Gröschel, A. Walther, T. I. Löbling, J. Schmelz, A. Hanisch, H. Schmalz and A. H. E. Müller, J. Am. Chem. Soc., 2012, 134, 13850-13860.

70 P. Hiekkataipale, T. I. Löbling, M. Poutanen, A. Priimagi, V. Abetz, O. Ikkala and A. H. Gröschel, Polymer, 2016, 107, $457 \mathrm{e} 466$.

71 K. Kawamoto, M. J. Zhong, K. R. Gadelrab, L.-C. Cheng, C. A. Ross, A. Alexander-Katz and J. A. Johnson, J. Am. Chem. Soc., 2016, 138, 11501-11504.

72 M. D. McConnell, M. J. Kraeutler, S. Yang and R. J. Composto, Nano Lett., 2010, 10, 603-609.

73 A. G. Skirtach, D. G. Kurth and H. Möhwald, Appl. Phys. Lett., 2009, 94, 093106.

74 X. Y. Ling, I. Y. Phang, C. Acikgoz, M. D. Yilmaz, M. A. Hempenius, G. J. Vancso and J. Huskens, Angew. Chem., Int. Ed., 2009, 48, 7677-7682.

75 S. Jiang and S. Granick, Langmuir, 2008, 24, 2438-2445.

76 L. Hong, S. Jiang and S. Granick, Langmuir, 2006, 22, 9495-9499.

77 T. M. Ruhland, H. S. McKenzie, T. S. Skelhon, S. A. F. Bon, A. Walther and A. H. E. Muller, Polymer, 2015, 79, 299308.

78 E. Sharifzadeh, M. Salami-Kalajahi, M. S. Hosseini and M. K. R. Aghjeh, Colloids Surf., A, 2016, 506, 56-62.

79 A. Zenerino, C. Peyratout and A. Aimable, J. Colloid Interface Sci., 2015, 450, 174-181.

80 W. L. Li, X. J. Cai, S. H. Ma, X. H. Zhan, F. Lan, Y. Wu and Z. W. Gu, RSC Adv., 2016, 6, 40450-40458.

81 Y. H. Wang, C. L. Zhang, C. Tang, J. Li, K. Shen, J. G. Liu, X. Z. Qu, J. Li, Q. Wang and Z. Z. Yang, Macromolecules, 2011, 44, 3787-3794.

82 R. H. Deng, H. Li, J. T. Zhu, B. H. Li, F. X. Liang, F. Jia, X. Z. Qu and Z. Z. Yang, Macromolecules, 2016, 49, 13621368.

83 T. Tanaka, M. Okayama, Y. Kitayama, Y. Kagawa and M. Okubo, Langmuir, 2010, 26, 7843-7847.

84 H. Ahmad, N. Saito, Y. Kagawa and M. Okubo, Langmuir, 2008, 24, 688-691.

85 N. Yamashita, N. Konishi, T. Tanaka and M. Okubo, Langmuir, 2012, 28, 12886-12892.

86 T. Yamagami, Y. Kitayama and M. Okubo, Langmuir, 2014, 30, 7823-7832.

87 C. C. Lin, C. W. Liao, Y. C. Chao and C. S. Kuo, ACS Appl. Mater. Interfaces, 2010, 2, 3185-3191.

88 C. C. Ho, W. S. Chen, T. Y. Shie, J. N. Lin and C. S. Kuo, Langmuir, 2008, 24, 5663-5666.

89 Q. Yang, M. H. de Vries, F. Picchioni and K. Loos, Nanoscale, 2013, 5, 10420-10427.
90 Q. Yang, Q. Xu and K. Loos, Macromolecules, 2015, 48, 1786-1794.

91 H. Yabu, K. Koike, K. Motoyoshi, T. Higuchi and M. Shimomura, Macromol. Rapid Commun., 2010, 31, 1267-1271.

92 H. Yabu, K. Motoyoshi, T. Higuchi and M. Shimomura, Phys. Chem. Chem. Phys., 2010, 12, 11944-11947.

93 T. Arita, M. Kanahara, K. Motoyoshi, K. Koike, T. Higuchi and H. Yabu, J. Mater. Chem. C, 2013, 1, 207-212.

94 H. Yabu, M. Kanahara, M. Shimomura, T. Arita, K. Harano, E. Nakamura, T. Higuchi and H. Jinnai, ACS Appl. Mater. Interfaces, 2013, 5, 3262-3266.

95 H. Yabu, H. Ohshima and Y. Saito, ACS Appl. Mater. Interfaces, 2014, 6, 18122-18128.

96 J. Yan, M. Bloom, S. C. Bae, E. Luijten and S. Granick, Nature, 2012, 491, 578-581.

97 J. Yan, K. Chaudhary, S. C. Bae, J. A. Lewis and S. Granick, Nat. Commun., 2013, 4, 1516.

98 J. Yan, S. C. Bae and S. Granick, Adv. Mater., 2015, 27, 874-879.

99 J. Yan, S. C. Bae and S. Granick, Soft Matter, 2015, 11, 147-153.

100 A. Ruditskiy, B. Ren and I. Kretzschmar, Soft Matter, 2013, 9, 9174-9181.

101 B. Ren and I. Kretzschmar, Langmuir, 2013, 29, 1477914786.

102 B. Ren, A. Ruditskiy, J. H. Song and I. Kretzschmar, Langmuir, 2012, 28, 1149-1156.

103 S. Sacanna, L. Rossi and D. J. Pine, J. Am. Chem. Soc., 2012, 134, 6112-6115.

104 B. Bharti and O. D. Velev, Langmuir, 2015, 31, 7897-7908.

105 P. C. Song, Y. F. Wang, Y. Wang, A. D. Hollingsworth, M. Weck, D. J. Pine and M. D. Ward, J. Am. Chem. Soc., 2015, 137, 3069-3075.

106 J. L. Cheng, J. P. He, C. X. Li and Y. L. Yang, Chem. Mater., 2008, 20, 4224-4230.

107 Y. Z. Wang, D. Q. Fan, J. P. He and Y. L. Yang, Colloid Polym. Sci., 2011, 289, 1885-1894.

108 P. G. De Gennes, Rev. Mod. Phys., 1992, 64, 645-648.

109 J. Faria, M. P. Ruiz and D. E. Resasco, Adv. Synth. Catal., 2010, 352, 2359-2364.

110 D. J. Cole-Hamilton, Science, 2010, 327, 41-42.

111 W. Cao, R. Huang, W. Qi, R. Su and Z. He, ACS Appl. Mater. Interfaces, 2015, 7, 465-473.

112 C. Wu, S. Ba, M. B. Ansorge-Schumacher and D. Wang, Adv. Mater., 2011, 23, 5694-5699.

113 B. P. Binks and P. D. I. Fletcher, Langmuir, 2001, 17, 47084710.

114 M. A. Fernandez-Rodriguez, J. Ramos, L. Isa, M. A. Rodriguez-Valverde, M. A. Cabrerizo-Vilchez and R. Hidalgo-Alvarez, Langmuir, 2015, 31, 8818-8823.

115 A. Walther, M. Hoffmann and A. H. E. Müller, Angew. Chem., Int. Ed., 2008, 47, 711-714.

116 M. A. Fernandez-Rodriguez, M. A. Rodriguez-Valverde, M. A. Cabrerizo-Vilchez and R. Hidalgo-Alvarez, $A d v$. Colloid Interface Sci., 2016, 233, 240-254. 
117 H. Rezvantalab and S. Shojaei-Zadeh, ACS Nano, 2016, 10, 5354-5361.

118 Q. G. Xie, G. B. Davies and J. Harting, Soft Matter, 2016, 12, 6566-6574.

119 E. Passas-Lagos and F. Schüth, Langmuir, 2015, 31, 77497757.

120 J. Lenis, S. Razavi, K. D. Cao, B. H. Lin, K. Y. C. Lee, R. S. Tu and I. Kretzschmar, J. Am. Chem. Soc., 2015, 137, 15370-15373.

121 D. L. Wu, J. W. Chew and A. Honciuc, Langmuir, 2016, 32, 6376-6386.

122 H. Rezvantalab, G. Drazer and S. Shojaei-Zadeh, J. Chem. Phys., 2015, 142, 014701.

123 H.-M. Gao, Z.-Y. Lu, H. Liu, Z.-Y. Sun and L.-J. An, J. Chem. Phys., 2014, 141, 134907.

124 X. C. Luu, J. Yu and A. Striolo, J. Phys. Chem. B, 2013, 117, 13922-13939.

125 X. C. Luu and A. Striolo, J. Phys. Chem. B, 2014, 118, 13737-13743.

126 D. L. Cheung and S. A. F. Bon, Soft Matter, 2009, 5, 39693976.

127 T. Tanaka, M. Okayama, H. Minami and M. Okubo, Langmuir, 2010, 26, 11732-11736.

128 Q. G. Xie, G. B. Davies, F. Günther and J. Harting, Soft Matter, 2015, 11, 3581-3588.

129 C. E. Estridge and A. Jayaraman, ACS Macro Lett., 2015, 4, 155-159.

130 T. Nakano, D. Kawaguchi and Y. Matsushita, J. Am. Chem. Soc., 2013, 135, 6798-6801.

131 C. W. Huang, J. P. Gao, W. Yu and C. X. Zhou, Macromolecules, 2012, 45, 8420-8429.

132 M. X. Huang, Z. Q. Li and H. X. Guo, Soft Matter, 2012, 8, 6834-6845.

133 M. X. Huang and H. X. Guo, Soft Matter, 2013, 9, 73567368.

134 A. Krekhov, V. Weith and W. Zimmermann, Phys. Rev. E: Stat. Phys., Plasmas, Fluids, Relat. Interdiscip. Top., 2013, 88, 040302.

135 N. Virgilio and B. D. Favis, Macromolecules, 2011, 44, 5850-5856.

136 A. Walther, K. Matussek and A. H. E. Muller, ACS Nano, 2008, 2, 1167-1178.

137 S. Weiss, D. Hirsemann, B. Biersack, M. Ziadeh, A. H. E. Müller and J. Breu, Polymer, 2013, 54, 13881396.

138 R. Bahrami, T. I. Löbling, A. H. Gröschel, H. Schmalz, A. H. E. Müller and V. Altstädt, ACS Nano, 2014, 8, 1004810056.
139 S. Barwinkel, R. Bahrami, T. I. Lobling, H. Schmalz, A. H. E. Muller and V. Altstadt, Adv. Eng. Mater., 2016, 18, 814-825.

140 K. C. Bryson, T. I. Löbling, A. H. E. Müller, T. P. Russell and R. C. Hayward, Macromolecules, 2015, 48, 4220-4227.

141 H. T. Wang, Z. Fu, W. Y. Dong, Y. J. Li and J. Y. Li, J. Phys. Chem. B, 2016, 120, 9240-9252.

142 H. T. Wang, W. Y. Dong and Y. J. Li, ACS Macro Lett., 2015, 4, 1398-1403.

143 T. Parpaite, B. Otazaghine, A. S. Caro, A. Taguet, R. Sonnier and J. M. Lopez-Cuesta, Polymer, 2016, 90, 34-44.

144 H. R. Nie, C. Zhang, Y. W. Liu and A. H. He, Macromolecules, 2016, 49, 2238-2244.

145 Z. Y. Liu, R. H. Guo, G. X. Xu, Z. H. Huang and L.-T. Yan, Nano Lett., 2014, 14, 6910-6916.

146 B. J. Kim, J. Bang, C. J. Hawker and E. J. Kramer, Macromolecules, 2006, 39, 4108-4114.

147 B. J. Kim, G. H. Fredrickson, J. Bang, C. J. Hawker and E. J. Kramer, Macromolecules, 2009, 42, 6193-6201.

148 B. J. Kim, G. H. Fredrickson and E. J. Kramer, Macromolecules, 2008, 41, 436-447.

149 J. J. Chiu, B. J. Kim, E. J. Kramer and D. J. Pine, J. Am. Chem. Soc., 2005, 127, 5036-5037.

150 B. J. Kim, J. Bang, C. J. Hawker, J. J. Chiu, D. J. Pine, S. G. Jang, S.-M. Yang and E. J. Kramer, Langmuir, 2007, 23, 12693-12703.

151 J. U. Kim and M. W. Matsen, Phys. Rev. Lett., 2009, 102, 078303.

152 S. Kim, T.-H. Kim, J. Huh, J. Bang and S.-H. Choi, ACS Macro Lett., 2015, 4, 417-421.

153 Q. F. Li, J. B. He, E. Glogowski, X. F. Li, J. Wang, T. Emrick and T. P. Russell, Adv. Mater., 2008, 20, 1462-1466.

154 M. K. Mayeda, W.-F. Kuan, W.-S. Young, J. A. Lauterbach and T. H. Epps III, Chem. Mater., 2012, 24, 2627-2634.

155 L. Q. Wang, J. P. Lin and X. M. Zhu, RSC Adv., 2012, 2, 12870-12878.

156 M. R. Bockstaller, Y. Lapetnikov, S. Margel and E. L. Thomas, J. Am. Chem. Soc., 2003, 125, 5276-5277.

157 C. T. Lo, Y. C. Chang, S. C. Wu and C. L. Lee, Colloids Surf., A, 2010, 368, 6-12.

158 R. B. Thompson, V. V. Ginzburg, M. W. Matsen and A. C. Balazs, Science, 2001, 292, 2469-2472.

159 L.-T. Yan, N. Popp, S.-K. Ghosh and A. Böker, ACS Nano, 2010, 4, 913-920.

160 B. J. Dong, R. H. Guo and L.-T. Yan, Macromolecules, 2014, 47, 4369-4379.

161 B. J. Dong, Z. H. Huang, H. L. Chen and L.-T. Yan, Macromolecules, 2015, 48, 5385-5393. 